\title{
Efeitos de Diferentes Formulações Comerciais de Glyphosate SOBRE ESTIRPES DE Bradyrhizobium ${ }^{1}$
}

\author{
Effects of Different Glyphosate Commercial Formulations on Bradyrhizobium Strains
}

\author{
SANTOS, J.B. ${ }^{2}$, JACQUES, R.J.S. ${ }^{3}$, PROCÓPIO, S.O. ${ }^{4}$, KASUYA, M.C.M. ${ }^{5}$, SILVA, A.A. ${ }^{6}$ e \\ SANTOS, E.A. ${ }^{7}$
}

\begin{abstract}
RESUMO - O objetivo deste trabalho foi avaliar efeitos de formulações comerciais de glyphosate sobre estirpes de Bradyrhizobium, em condições de laboratório. As formulações foram aplicadas na concentração de $43,2 \mu \mathrm{g} \mathrm{L}^{-1}$ do equivalente ácido. As bactérias foram inoculadas em meio de cultura à base de manitol e extrato de levedura (YM). O efeito do herbicida no crescimento das estirpes de Bradyrhizobium foi avaliado mediante leitura da densidade ótica em espectrofotômetro. Avaliou-se o crescimento das estirpes de $B$. japonicum SEMIA 5079 e de B. elkanii SEMIA 5019 e SEMIA 587 sob efeito de nove formulações de glyphosate: Zapp Qi ${ }^{\circledR}$, Roundup ${ }^{\circledR}$, Roundup Multiação ${ }^{\circledR}$, Roundup Transorb ${ }^{\circledR}$, Roundup WG ${ }^{\circledR}$, Trop $\AA$, Agrisato ${ }^{\circledR}$, glyphosate técnico [padrão de N-(phosphonomethyl) glycina] e controle sem adição de herbicida (testemunha para as estirpes). Foram utilizadas seis repetições. Confeccionaram-se curvas de crescimento para cada estirpe. Pelos resultados, pôde-se observar que todas as formulações de glyphosate causaram efeitos diferenciados sobre as estirpes de Bradyrhizobium SEMIA 5019, SEMIA 5079 e SEMIA 587. Constatou-se que a formulação Zapp Qi foi a menos tóxica às estirpes de Bradyrhizobium avaliadas. A maior toxicidade foi observada para Roundup Transorb, que provocou reduções no crescimento acima de $94 \%$ para todas as estirpes de Bradyrhizobium estudadas. Não se observou correlação entre o tipo de sal - isopropilamina, amônio ou potássico, presentes na formulação herbicida - e o grau de inibição no crescimento das estirpes. SEMIA 587 foi a estirpe menos tolerante à maioria das formulações testadas, porém SEMIA 5019 foi a mais sensível ao glyphosate padrão, sem adição de sais ou de outros aditivos.
\end{abstract}

Palavras-chave: fixação biológica de $\mathrm{N}_{2}$, herbicida, rizóbio, soja transgênica.

\begin{abstract}
This work aimed to evaluate the effects of glyphosate commercial formulations on Bradyrhizobium strains under laboratory conditions. The formulations were applied in the concentration of $43.2 \mu \mathrm{g} \mathrm{L}^{-1}$ of the a.e. and the strains were inoculated in yeast extract manitol (YM). Herbicide effect on the growth of the Bradyrhizobium strains was assessed by optic density reading in a spectrophotometer. Twenty seven treatments arranged in a factorial design were evaluated and consisted of one strain of B. japonicum: SEMIA 5079; and two strains of B. elkanii: SEMIA 5019 and SEMIA 587, under the effect of nine glyphosate formulations: Zapp ${ }_{G I}{ }^{\circledR}$, Roundup ${ }^{\circledR}$, Roundup Multiação®, Roundup Transorb ${ }^{\circledR}$, Roundup $W G \AA$, Trop $\AA$, Agrisato ${ }^{\circledR}$, technical glyphosate [ $\mathrm{N}$-(phosphonomethyl) glycine] and control without herbicide addition (as the strain control treatment), with six replications. A growth curve was established for each strain. It could be observed that the different glyphosate formulations Zapp Qi, Roundup, Roundup Multiação, Roundup transorb, Roundup WG, Trop and Agrisato caused differentiated effects on the strains of Bradyrhizobium SEMIA 5019, SEMIA 5079 and SEMIA 587. It was verified that the Zapp Qi formulation was the least toxic to the strains. The highest toxicity was observed for Roundup Transorb, which reduced growth over 94\% for all the strains assessed. Correlation was not
\end{abstract}

Recebido para publicação em 14.5.2003 e na forma revisada em 5.3.2004.

2 Mestrando, Dep. de Fitotecnia da Universidade Federal de Viçosa-UFV, 36570-000 Viçosa-MG, <jbarbosasantos@ yahoo.com.br>. ${ }^{3}$ Doutorando, Dep. de Solos - UFRGS, <rjsjacques@ zipmail.com.br>. ${ }^{4}$ Prof. do Dep. de Fitossanidade da Universidade Federal de Pelotas - UFPEL, 96010-900 Pelotas-RS; ${ }^{5}$ Professora, Dep. de Microbiologia - UFV, <mkasuya@ufv.br>; ${ }^{6}$ Professor, Dep. de Fitotecnia - UFV, <aasilva@ ufv.br>; ${ }^{7}$ Acadêmico de Agronomia, Dep. de Fitotecnia - UFV.

Planta Daninha, Viçosa-MG, v.22, n.2, p.293-299, 2004 
observed among the type of salt, isopropylamine, ammonium or potassic, present in the formulation herbicides, and the toxicity degree to the strains. The strain SEMIA 587 was the least tolerant to most formulations while SEMIA 5019 was the most sensitive to the control treatment $\mathrm{N}$ (phosphonomethyl) glycine, without salts or other additives.

Key words: biological fixation of $\mathrm{N}_{2}$, herbicide, rhizobium, transgenic soybean.

\section{INTRODUÇÃO}

O glyphosate é o herbicida de maior participação no mercado mundial de herbicidas, caracterizando-se pelo amplo espectro de ação e por não ser seletivo à cultura da soja. Existem, atualmente, mais de 90 marcas comerciais com esse ingrediente ativo no mundo (Heap, 1997). No entanto, esse herbicida poderá ser utilizado no Brasil diretamente na cultura da soja, caso haja introdução de cultivares resistentes a essas moléculas. Por inibir a síntese da enzima 5-enolpiruvilshiquimato-3-fosfato sintase (EPSPs), a molécula de glyphosate bloqueia a sintese de aminoácidos aromáticos. Cultivares de soja resistentes ao glyphosate contêm a enzima EPSPs insensivel ao herbicida, todavia as bactérias fixadoras de $\mathrm{N}_{2}$, associadas ao sistema radicular da soja, do gênero Bradyrhizobium, não apresentam essa insensibilidade enzimática, podendo o glyphosate interferir na interação simbiótica. Segundo Gonzalez et al. (1996), o risco de intoxicação de herbicidas sobre os microrganismos é maior quando os produtos inibem processos bioquímicos comuns entre as plantas e os microrganismos.

Em outros países, foi constatado que o glyphosate provocou intoxicação em estirpes de rizóbios ou prejudicou a nodulação de leguminosas, inclusive da soja (Mallik \& Tesfai, 1985; Schuls et al., 1985; Eberbach \& Douglas, 1989; Moorman et al., 1992; Haugland, 1994; Hernandez et al., 1999; King et al., 2001). Entretanto, em razão da grande variabilidade nas respostas de estirpes de rizóbio à presença de herbicidas (Kapusta \& Rouwenhorst, 1973; Alagavadi \& Reddy, 1986; Kishinevsky et al., 1988; Delanay et al., 1995), é importante avaliar a natureza e a intensidade de resposta das principais estirpes utilizadas no Brasil.

Segundo Malkones (2000), os aditivos presentes na formulação dos agroquímicos podem afetar os microrganismos e, em certos casos, até modificar o efeito do agroquímico, porém poucos trabalhos foram realizados sobre $o$ assunto. Berner et al. (1991) mencionaram que aplicações de glyphosate, em formulações com ou sem surfatante, inibiram o crescimento micelial de Calonectria crotalariae.

Objetivou-se neste trabalho avaliar a toxicidade das principais formulações de glyphosate comercializadas no Brasil sobre três estirpes comerciais do gênero Bradyrhizobium.

\section{MATERIAL E MÉTODOS}

O experimento foi realizado no Laboratório de Associações Biológicas (FBN)/BIOAGRO, pertencente ao Departamento de Microbiologia da Universidade Federal de Viçosa, Viçosa MG.

As soluções-estoque das formulações de glyphosate foram preparadas mediante a mistura do produto comercial com água destilada e deionizada, sendo posteriormente esterilizadas por filtração (filtro Milipore 0,25 $\mu \mathrm{m}$ ) em condições assépticas.

As estirpes de Bradyrhizobium foram obtidas da Coleção de Bactérias Diazotróficas do Centro Nacional de Pesquisa da EMBRAPA/ Agrobiologia, sendo as culturas estocadas em frascos de ágar inclinado com meio à base de extrato de levedura e manitol como fonte de carbono, meio $\mathrm{YM}$, composto, em $\mathrm{g} \mathrm{L}^{-1}$, por: manitol, 10; $\mathrm{K}_{2} \mathrm{HPO}_{4}, 0,05 ; \mathrm{MgSO}_{4}, 0,02 ; \mathrm{NaCl}$, 0,01; extrato de levedura, 0,5; ágar, 15; e pH ajustado em 6,8 , à temperatura de $4{ }^{\circ} \mathrm{C}$. A ativação das estirpes foi feita mediante inoculação em frascos erlenmeyer de $125 \mathrm{~mL}$, contendo $50 \mathrm{~mL}$ de meio YM líquido, e incubação em agitador rotatório a $125 \mathrm{rpm}$ e temperatura de $25{ }^{\circ} \mathrm{C}$. Uma alíquota de $15 \mu \mathrm{L}$ das estirpes ativadas foi inoculada em placas de ELISA de 96 células com capacidade volumétrica de $300 \mu \mathrm{L}$, contendo a mistura estéril 
Tabela 1 - Formulações, fabricante e composição dos produtos comerciais de glyphosate utilizados

\begin{tabular}{|l|l|l|l|}
\hline Formulações comerciais* & Fabricante & Composição & Formulação (expressa em equivalente ácido) \\
\hline Agrisato & Alkagro & Sal de isopropilamina & Concentrado solúvel $\left(360 \mathrm{~g} \mathrm{~L}^{-1}\right)$ \\
\hline Roundup (original) & Monsanto & Sal de isopropilamina & Concentrado solúvel $\left(360 \mathrm{~g} \mathrm{~L}^{-1}\right)$ \\
\hline Roundup Multiação & Monsanto & Sal de amônio & Grânulos autodispersíveis em água $\left(720 \mathrm{~g} \mathrm{~kg}^{-1}\right)$ \\
\hline Roundup Transorb & Monsanto & Sal de isopropilamina & Concentrado solúvel $\left(480 \mathrm{~g} \mathrm{~L}^{-1}\right)$ \\
\hline Roundup WG & Monsanto & Sal de amônio & Grânulos autodispersíveis em água $\left(720 \mathrm{~g} \mathrm{~kg}^{-1}\right)$ \\
\hline Trop & Milênia & Sal de isopropilamina & Concentrado solúvel $\left(360 \mathrm{~g} \mathrm{~L}^{-1}\right)$ \\
\hline Zapp Qi & Syngenta & Sal potássico & Concentrado solúvel $\left(500 \mathrm{~g} \mathrm{~L}^{-1}\right)$ \\
\hline
\end{tabular}

* Marca registrada do fabricante ${ }^{\circledR}$.

de $180 \mu \mathrm{L}$ de meio YM líquido (1,33 vez concentrado) e $60 \mu \mathrm{L}$ da solução do herbicida, para cada tratamento, sendo imediatamente colocadas em câmara de incubação a $25^{\circ} \mathrm{C}$ e no escuro. O crescimento das estirpes foi avaliado pela leitura da densidade ótica a $560 \mathrm{~nm}$ em espectrofotômetro (Titertek Multiskan Plus MKII, New York, USA), em intervalos de quatro horas nas primeiras 24 horas e, após, em intervalos de 12 horas até a estabilização do crescimento.

O delineamento experimental utilizado foi o inteiramente casualizado em esquema fatorial $3 \times 9$, totalizando 27 tratamentos, sendo estes a combinação de duas estirpes de $B$. elkanii SEMIA 587 e SEMIA 5019, uma de B. japonicum SEMIA 5079 e nove formulações de glyphosate: Zapp Qi, Roundup, Roundup Multiação, Roundup Transorb, Roundup WG, Trop, Agrisato, PMG [padrão N-(phosphonomethyl) glycina com 99\% de pureza] e o controle sem adição de herbicida, com seis repetições. Todas as formulações foram aplicadas na concentração de $43,2 \mu \mathrm{g} \mathrm{L}^{-1}$, correspondente a $8,64 \mathrm{~kg} \mathrm{ha}^{-1}$ de glyphosate, considerando área por área.

Os resultados obtidos, após 122 horas de incubação, foram submetidos à análise de variância, e as médias, comparadas pelo teste de Tukey a $5 \%$ de probabilidade. Também se utilizou análise de regressão para traçar curvas de crescimento bacteriano durante o período de avaliação, para os diferentes tratamentos avaliados.

\section{RESULTADOS E DISCUSSÃO}

As sete marcas comerciais testadas afetaram o desenvolvimento das estirpes; todavia, entre as formulações houve diferença no grau de intoxicação (Figuras 1, 2 e 3 e Tabela 3).

A estirpe SEMIA 5019 foi a mais sensivel à molécula pura do glyphosate $(P M G)$ (Figura 1 e Tabela 3). Para esta estirpe, as inibições máximas de crescimento medidas pelo aumento da densidade ótica (DO), observadas entre os tratamentos, variaram desde $25,87 \%$, para Zapp Qi, até $97,82 \%$, para R. Transorb (Tabela 3). A formulação Zapp Qi (mais tolerada por esta estirpe) foi menos tóxica do que o padrão $\mathrm{N}$-(phosphonomethyl) glycina $(P M G)$, utilizado como controle para os herbicidas (Figura 1 e Tabela 3). As formulações Roundup (original), Trop, Agrisato e Roundup Multiação foram semelhantes quanto à toxicidade a esta estirpe, proporcionando, em média, inibição do crescimento de $56 \%$ em relação ao controle (Figura 1 e Tabela 3). A formulação Roundup WG reduziu o crescimento desta estirpe em mais de $65 \%$, comparado ao controle sem adição de herbicida. Roundup Transorb afetou drasticamente esta estirpe (Figura 1 e Tabela 3). Pelas equações das curvas de regressão, observou-se ausência de crescimento desta estirpe sob efeito de R. Transorb (Tabela 2).

A estirpe SEMIA 5079 apresentou, quando submetida à formulação Trop, crescimento similar ao do controle (Figura 2 e Tabela 3). As formulações Zapp Qi e Roundup Multiação inibiram o crescimento desta estirpe em 22,79 e $26,48 \%$, respectivamente, em relação ao controle sem adição de herbicida; contudo, 122 horas após a inoculação, esses valores não diferiram significativamente do controle herbicida $P M G$ (16,64\% de inibição) (Tabela 3). Conforme pode ser observado na Figura 2, esta estirpe se mostrou altamente sensivel às 


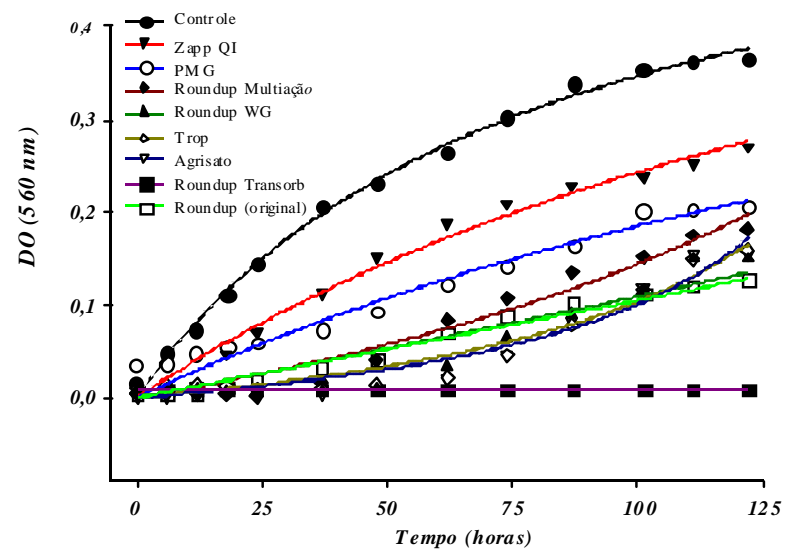

Figura 1 - Efeito de diferentes formulações de glyphosate (43,2 $\mu \mathrm{g} \mathrm{L}^{-1}$ de e.a.) sobre o crescimento da estirpe de B. elkanii SEMIA 5019 - média de seis repetições.

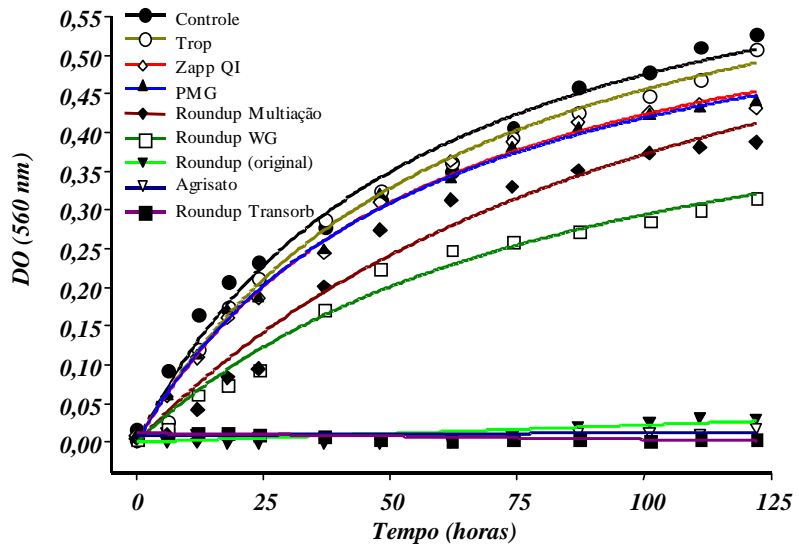

Figura 2 - Efeito de diferentes formulações de glyphosate (43,2 $\mu \mathrm{g} \mathrm{L}^{-1}$ de e.a.) sobre o crescimento da estirpe de B. japonicum SEMIA 5079 - média de seis repetições.

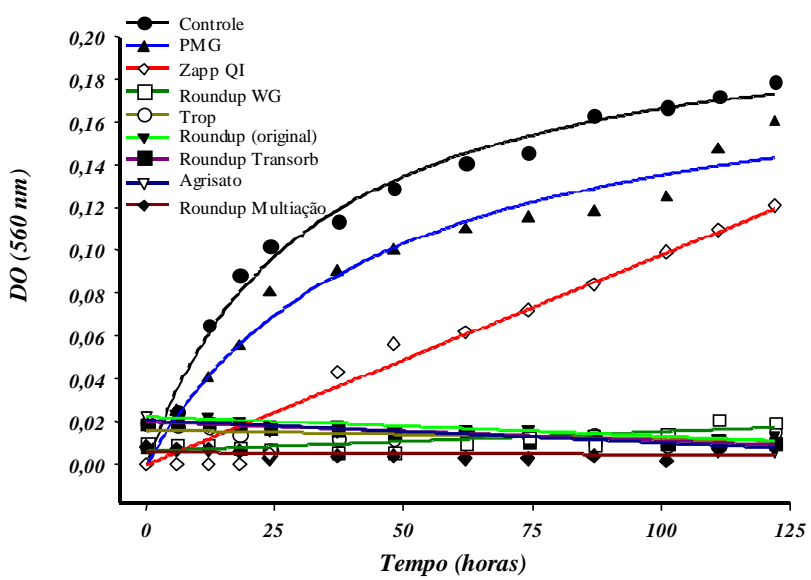

Figura 3 - Efeito de diferentes formulações de glyphosate (43,2 $\mu \mathrm{g} \mathrm{L}^{-1}$ de e.a.) sobre o crescimento da estirpe de B. elkanii SEMIA 587 - média de seis repetições. formulações Roundup (original), Agrisato e Roundup Transorb, visto estas terem reduzido o crescimento em mais de $90 \%$. Dessa forma, SEMIA 5079 sob efeito de tais herbicidas, na concentração testada, em condições de laboratório, apresentou desenvolvimento quase nulo. Novamente Roundup Transorb foi a formulação mais tóxica, sendo observada inibição no crescimento acima de $99 \%$, em relação ao controle (Tabela 3), contudo não diferiu das formulações Roundup (original) e Agrisato (Figura 2 e Tabela 3).

Tabela 2 - Equações das curvas de regressão relacionando densidade ótica (DO) e tempo de avaliação (horas), para diferentes formulações de glyphosate testadas sobre o crescimento das estirpes de B. elkanii SEMIA 5019 e SEMIA 587 e B. japonicum SEMIA 5079

\begin{tabular}{|c|c|c|}
\hline Tratamento & Equação* & \begin{tabular}{|c}
$\begin{array}{c}\text { Coeficiente de } \\
\text { determinação } \\
\left(\mathrm{R}^{2}\right)\end{array}$ \\
\end{tabular} \\
\hline \multicolumn{3}{|c|}{ SEMIA 5019} \\
\hline Controle (sem herbicida) & $\hat{\mathrm{Y}}=0,0079 \mathrm{x} /(1+0,0128 \mathrm{x})$ & 0,99 \\
\hline PMG** & $\hat{Y}=0,0026 x /(1+0,0039 x)$ & 0,96 \\
\hline Zapp Qi & $\begin{array}{l}\hat{Y}=0,0037 x /(1+0,0050 x) \\
0\end{array}$ & 0,98 \\
\hline Agrisato & $\hat{Y}=0,0004 x /(1-0,056 x)$ & 0,99 \\
\hline Roundup Transorb & $\hat{\mathrm{Y}}=(0,007-0,0008 \mathrm{x})$ & 0,86 \\
\hline Roundup $W G$ & $\hat{\mathrm{Y}}=0,0010 \mathrm{x} /(1-0,0005 \mathrm{x})$ & 0,98 \\
\hline Roundup Multiação & $\hat{Y}=0,0010 x /(1-0,0033 x)$ & 0,95 \\
\hline Roundup (original) & $\hat{\mathrm{Y}}=0,0010 \mathrm{x} /(1-0,0002 \mathrm{x})$ & 0,97 \\
\hline Trop & $\hat{\mathrm{Y}}=0,0005 \mathrm{x} /(1-0,0051 \mathrm{x})$ & 0,95 \\
\hline \multicolumn{3}{|c|}{ SEMIA 5079} \\
\hline Controle (sem herbicida) & $\hat{Y}=0,7401 x /(55,8535+x)$ & $\begin{array}{l}0,98 \\
0,9\end{array}$ \\
\hline $\mathrm{PMG}^{* *}$ & $\hat{\mathrm{Y}}=0,6505 \mathrm{x} /(55,1917+\mathrm{x})$ & 0,99 \\
\hline Zapp Qi & $\hat{Y}=0,6673 x /(57,6099+x)$ & 0,99 \\
\hline Agrisato & $\hat{\mathrm{Y}}=(0,0114+0,0001 \mathrm{x})$ & 0,84 \\
\hline Roundup Transorb & $\hat{Y}=(0,0118-0,0005 x)$ & 0,80 \\
\hline Roundup $W G$ & $\begin{array}{l}\hat{Y}=0,5460 x /(85,6901+x) \\
0\end{array}$ & 0,98 \\
\hline Roundup. Multiação & $\hat{Y}=0,8037 x /(115,9907+x)$ & 0,97 \\
\hline Roundup (original) & $\hat{\mathrm{Y}}=(0,0119+0,0001 \mathrm{x})$ & 0,79 \\
\hline Trop & $\hat{Y}=0,7430 x /(63,0312+x)$ & 0,99 \\
\hline \multicolumn{3}{|c|}{ SEMIA 587} \\
\hline Controle (sem herbicida) & $\hat{Y}=0,2170 x /(30,5341+x)$ & 0,98 \\
\hline PMG** & $\hat{Y}=0,1963 x /(45,1041+x)$ & 0,97 \\
\hline Zapp Qi & $\hat{Y}=1421,23 x /(1,45 E 6+x)$ & 0,95 \\
\hline Agrisato & $\hat{\mathrm{Y}}=(0,0203-0,0001 \mathrm{x})$ & 0,82 \\
\hline Roundup Transorb & $\hat{Y}=(0,0195-0,0001 x)$ & 0,93 \\
\hline Roundup $W G$ & $\hat{\mathrm{Y}}=(0,0062+0,0001 \mathrm{x})$ & 0,85 \\
\hline Roundup Multiação & $\hat{\mathrm{Y}}=(0,0056-0,00001 \mathrm{x})$ & 0,86 \\
\hline Roundup (original) & $\hat{\mathrm{Y}}=(0,0223-0,0001 \mathrm{x})$ & 0,91 \\
\hline Trop & $\hat{\mathrm{Y}}=(0,0157-0,00001 \mathrm{x})$ & 0,91 \\
\hline
\end{tabular}

* Significativo pelo teste $\mathrm{t}(\mathrm{P}<0,05)$ e ** produto puro padrão de $\mathrm{N}$-(phosphonomethyl) glycina com $99 \%$ de pureza. 
Tabela 3 - Efeito de diferentes formulações de glyphosate (43,2 $\left.\mu \mathrm{g} \mathrm{L} \mathrm{L}^{-1}\right)$ sobre o crescimento de estirpes de Bradyrhizobium, medido por meio do aumento da densidade ótica (DO), 122 horas após inoculação

\begin{tabular}{|l|c|c|r|}
\hline \multirow{2}{*}{ Tratamento } & \multicolumn{3}{|c|}{ DO 560 nm (\%) } \\
\cline { 2 - 4 } & SEMIA 5019 & SEMIA 5079 & SEMIA 587 \\
\hline Controle & $100,00 \mathrm{a}$ A & $100,00 \mathrm{a}$ A & $100,00 \mathrm{a}$ A \\
Trop & $40,71 \mathrm{de} \mathrm{B}$ & $96,27 \mathrm{ab} \mathrm{A}$ & $22,72 \mathrm{c} \mathrm{C}$ \\
Roundup (original) & $43,81 \mathrm{de} \mathrm{A}$ & $6,09 \mathrm{~d} \mathrm{~B}$ & $7,45 \mathrm{~d} \mathrm{~B}$ \\
Agrisato & $42,21 \mathrm{de} \mathrm{A}$ & $3,63 \mathrm{~d} \mathrm{~B}$ & $3,82 \mathrm{~d} \mathrm{~B}$ \\
Roundup Transorb & $2,18 \mathrm{~d} \mathrm{~A}$ & $0,79 \mathrm{~d} \mathrm{~A}$ & $5,59 \mathrm{~d} \mathrm{~A}$ \\
Roundup WG & $34,88 \mathrm{e} \mathrm{B}$ & $59,75 \mathrm{c} \mathrm{A}$ & $2,89 \mathrm{~d} \mathrm{C}$ \\
Roundup Multiação & $49,95 \mathrm{~cd} \mathrm{~B}$ & $73,52 \mathrm{~b} \mathrm{~A}$ & $1,44 \mathrm{~d} \mathrm{C}$ \\
Zapp Qi & $74,13 \mathrm{~b} \mathrm{AB}$ & $77,21 \mathrm{~b} \mathrm{~A}$ & $67,41 \mathrm{~b} \mathrm{~B}$ \\
PMG* & $56,47 \mathrm{c} \mathrm{B}$ & $83,36 \mathrm{~b} \mathrm{~A}$ & $89,11 \mathrm{a} \mathrm{A}$ \\
\hline
\end{tabular}

Médias seguidas de letras iguais, maiúscula na linha e minúscula na coluna, não diferem entre si pelo teste de Tukey a 5\% de probabilidade.

* Produto puro padrão de N-(phosphonomethyl) glycina com 99\% de pureza.

Com base nas equações referentes às curvas de regressão dos tratamentos, para SEMIA 5079 (Tabela 2), nota-se comportamento linear desta estirpe sob efeito das formulações Roundup (original), Agrisato e Roundup Transorb, inclusive com valores decrescentes para esta última.

SEMIA 587 foi, de maneira geral, a estirpe mais sensível às formulações do glyphosate testadas, visto que, entre estas, cinco impossibilitaram seu crescimento (Figura 3). Mesmo com o decréscimo no valor máximo de DO, foi possivel observar que, além do tratamento controle (sem adição de herbicida), a estirpe apresentou crescimento somente nas formulações Zapp Qi e PMG (controle herbicida) (Figura 3 e Tabela 3). O controle $P M G$, após 122 horas de incubação, não apresentou diferença quando comparado ao controle sem herbicida; já nesse mesmo período a formulação Zapp Qi inibiu em $32,59 \%$ o crescimento (Tabela 3). Por meio da análise das curvas de crescimento (Tabela 2), pôde-se constatar que as formulações Agrisato, Roundup Transorb, Roundup WG, Roundup Multiação e Roundup (original) reduziram de forma drástica o crescimento de SEMIA 587. Os dados ajustaram-se em equações do primeiro grau, evidenciando comportamento linear decrescente, exceto para Roundup WG (Tabela 2). A formulação Trop também resultou em alta inibição, alcançando valor próximo a $80 \%$ após 122 horas de incubação (Figura 3 e Tabela 3).

Haahtela et al. (1988) testaram o glyphosate puro e na formulação comercial Roundup sobre vários microrganismos e observaram que as concentrações entre 25 e $100 \mu \mathrm{g} \mathrm{L}^{-1}$ afetaram significativamente o crescimento de bactérias do gênero Enterobacter; entretanto, houve maior intoxicação quando elas foram tratadas com a formulação comercial na maior concentração.

Em relação aos sais que integram as formulações estudadas, pouca inferência se pode fazer na discussão dos resultados. O sal de isopropilamina compõe as formulações Agrisato, Roundup (original), Roundup Transorb e Trop, as quais apresentaram diferentes graus de intoxicação às estirpes e entre elas. O sal de amônio presente na composição de Roundup WG e Roundup Multiação não conferiu a estes o mesmo grau de intoxicação para as três estirpes (Tabela 2). No entanto, pode-se destacar que Zapp Qi, único que se apresenta com o sal potássico, foi a formulação que se manteve menos tóxica a todas as estirpes, sendo, para SEMIA 587, o único tratamento que se apresentou não-tóxico (Figura 3 e Tabela 2).

Com base nas curvas de regressão e comparação das médias depois de 122 horas de inoculação, pode-se estabelecer a seguinte ordem crescente de intoxicação dos herbicidas às estirpes: Zapp Qi $<P M G<$ Roundup Multiação $=$ Roundup $($ original $)=$ Agrisato $=$ Trop = Roundup WG $<$ Roundup Transorb, para SEMIA 5019; Trop $<P M G=$ Zapp Qi = Roundup Multiação $<$ Roundup WG $<$ Roundup (original) $=$ Agrisato $=$ Roundup Transorb, para SEMIA 5079; e $P M G<$ Zapp Qi < Trop < Roundup $($ original $)=$ Agrisato $=$ Roundup WG = Roundup Transorb, para SEMIA 587.

Entre as estirpes, a ordem crescente de tolerância foi a seguinte: SEMIA 587 < SEMIA 5019 < SEMIA 5079, para as formulações Trop, Roundup WG e Roundup Multiação; SEMIA 587 = SEMIA $5079<$ SEMIA 5019, para Agrisato e Roundup (original); SEMIA 5019 < SEMIA 5079 = SEMIA 587, para o controle herbicida $P M G$; e SEMIA 587 < SEMIA 5079, porém sem diferirem de SEMIA 5019, para 
Zapp Qi. A formulação Roundup Transorb causou elevado grau de intoxicação entre todas as estirpes.

A maior sensibilidade da estirpe SEMIA 587, em relação a SEMIA 5019, também foi constatada por Ortiz et al. (1989), quando crescida em meio contendo trifluralin. Arruda et al. (2001), trabalhando com herbicidas inibidores da enzima AHAS, em diferentes concentrações, observaram que eles afetaram drasticamente as estirpes de $B$. japonicum testadas, sendo estabelecidas diferentes ordens de tolerância para cada herbicida.

A redução do crescimento das estirpes de Bradyrhizobium na presença do equivalente ácido e das formulações comerciais de glyphosate provavelmente se deve à inibição da atividade da enzima EPSP sintase e à conseqüente redução da síntese de aminoácidos aromáticos nas células bacterianas. Moorman (1986) não observou redução do crescimento do Bradyrhizobium japonicum na presença de glyphosate quando aminoácidos aromáticos foram adicionados ao meio de cultura. É provável que os efeitos específicos das formulações comerciais de glyphosate no crescimento das estirpes sejam decorrentes da presença de diferentes substâncias químicas na formulação do herbicida, como solventes, surfatantes e agentes molhantes, que podem modificar o efeito do equivalente ácido do herbicida nos organismos (Kishinevsky et al., 1988; Malkones, 2000).

\section{AGRADECIMENTOS}

À empresa Syngenta Proteção de Cultivos, pelo apoio financeiro.

\section{LITERATURA CITADA}

ALAGAVADI, A. R.; REDDY, T. K. R. Effect of trifluralin on Rhizobium and its nodulation on groundnut. Pesticides, v. 20, p. 27-30, 1986.

ARRUDA, J. S.; LOPES, N. F.; MOURA, A. B. Behavior of Bradyrhizobium japonicum strains under different herbicide concentrations. Planta Daninha, Viçosa, v. 19, p. 111-117, 2001.

BERNER, D. K.; BERGGREN, G. T.; SNOW, J. P. Effects of glyphosate on Calonectia crotalariae and red crown rot of soybean. Plant Disease, v. 75, p. 809-813, 1991.
DELANAY, X. et al. Yield evaluation of a glyphosatetolerant soybean line after treatment with glyphosate. Crop Sci., v. 35, p. 1461-1467, 1995.

EBERBACH, P. L.; DOUGLAS, L. A. Herbicide effects on the growth and nodulation potential of Rhizobium trifolii with Trifolium subterraneum. Plant Soil, v. 119, p. 15-23, 1989.

EMPRESA BRASILEIRA DE PESQUISA AGROPECUÁRIA - EMBRAPA. Centro Nacional de Pesquisa de Arroz e Feijão. Manual de métodos empregados em estudos de Microbiologia Agrícola. Brasília, 1994. 542 p.

GONZALEZ, A.; GONZALEZ-MURUA, C.; ROYUELA, M. Influence of imazethapyr on Rhizobium growth and its symbiosis with Pea (Pisum sativum ). Weed Sci., v. 44, p. 31-37, 1996.

HAAHTELA, K.; KILPI, S.; KARI, K. Effects of phenoxy acid herbicides and glyphosate on nitrogenase activity (acetylene reduction) in root-associated Azospirillum, Enterobacter and Klebsiella. FEMS Microbiol. Lett., v. 53, p. 123-127, 1988.

HAUGLAND, E. Ecological consequences of chemical weed control. Norsk Landbruksf., v. 8, p. 1-13, 1994.

HEAP, I. M. The occurrence of herbicide resistant weeds world wide. Pestic. Sci., v. 51, p. 235-243, 1997.

HERNANDEZ, A.; GARCIA-PLAZAOLA, J. I.; BACERRIL, J. M. Glyphosate effects on phenolic metabolism of nodulated soybean (Glycine $\max$ L. Merr.). J. Agric. Food Chem., v. 47, p. 2920-2925, 1999.

KAPUSTA, G.; ROUWENHORST, D. L. Interaction of selected pesticides and Rhizobium japonicum in pure culture and under field conditions. Agron. J., v. 65, p. 112-115, 1973.

KING, C. A.; PURCELL, L. C.; VORIES, E. D. Plant growth and nitrogenase activity of glyphosate-tolerant soybean in response to foliar glyphosate applications. Agron. J.. v. 93, p. 176-186, 2001.

KISHINEVSKY, B. et al. Effects of some commercial herbicides on rhizobia and their symbiosis with peanuts. Weed Res., v. 28, p. 291-296, 1988.

MALLIK, M. A. B.; TESFAI, K. Pesticidal effect of soybean-rhizobia symbiosis. Plant Soil, v. 85, p. 33-41, 1985.

MALKONES, H. P. Comparison of the effects of differently formulated herbicides on soil microbial activities - a review. J. Plant Dis. Protect., v. 8, p. 781-789, 2000. 
MOORMAN, T. B. Effects of herbicides on the survival of Rhizobium japonicum strains. Weed Sci., v. 34, p. 628-633, 1986.

MOORMAN, T. B. et al. Production of hydroxybenzoic acids by Bradyrhizobium japonicum strains after treatment with glyphosate. J. Agric. Food Chem., v. 40, p. 289-293, 1992.
ORTIZ, S.; MUSUMECI, M. R.; TSAI, S. M. Efeito de alguns agrotóxicos na sobrevivência e na atividade respiratória de Rhizobium leguminosarum e Bradyrhizobium japonicum. Pesq. Agropec. Bras., v. 24, p. 663-667, 1989.

SCHULS, A.; KRUPER, A.; AMRHEIN, N. Differential sensitivity of bacterial S-enolpyruvyl shikimate-3phosphate synthases to the herbicide glyphosate. FEMS Microbiol. Lett., v. 28, p. 297-301, 1985. 